\title{
Nobiletin ameliorates hepatic ischemia and reperfusion injury through the activation of SIRT-1/FOXO3a-mediated autophagy and mitochondrial biogenesis
}

\author{
Theodomir Dusabimana ${ }^{1,2}$, So Ra Kim¹, Hye Jung Kim,2, Sang Won Park $\mathbb{B}^{1,2}$ and Hwajin Kim
}

\begin{abstract}
Hepatic ischemia and reperfusion injury are characterized by impaired autophagy, mitochondrial dysfunction, and subsequent compromise of cellular homeostasis following hepatic surgery or transplantation. Nobiletin, a natural flavonoid, is a beneficial antioxidant that possesses anti-inflammatory and anti-cancer activities. We investigated the effect of nobiletin on hepatic IR injury and described the underlying mechanisms. C57BL/6 mice were subjected to 60 min of partial hepatic ischemia, treated with nobiletin $(5 \mathrm{mg} / \mathrm{kg})$ or vehicle at the start of reperfusion, and killed at $5 \mathrm{~h}$ of reperfusion. Hepatic ischemia and reperfusion increased hepatocellular oxidative damage, inflammation, and cell death, but these changes were alleviated upon nobiletin treatment. Nobiletin increased the expression of proteins that control autophagy, mitochondrial dynamics, and biogenesis. Specifically, the SIRT-1/FOXO3a and PGC-1a pathways were activated by nobiletin. IR-induced AKT activation was associated with FOXO3a phosphorylation, which resulted in a significant reduction in the nuclear $\mathrm{FOXO}$ a levels and potentially attenuated autophagy-regulatory gene expression. Nobiletin increased FOXO3a expression and its nuclear translocation via the inhibition of AKT. Specific inhibition of SIRT-1 abolished the protective effect of nobiletin, causing decreased FOXO3a expression, followed by autophagy induction and decreased PGC-1a expression and mitochondrial dynamics. Taken together, our data indicate that SIRT-1 directly mediates the protective effect of nobiletin against hepatic ischemia and reperfusion injury. The activation of autophagy and mitochondrial function through the SIRT-1/FOXO3a and PGC-1a pathways indicate that nobiletin could have therapeutic potential for treating hepatic ischemia and reperfusion injury.
\end{abstract}

\section{Introduction}

Hepatic ischemia and reperfusion (IR) injury, caused by blood deprivation followed by reperfusion, occurs in various clinical settings, including hepatic resection surgery, transplantation, and shock. The pathophysiology of hepatic IR injury contributes to an increased rate of acute liver failure, graft rejection, and chronic hepatic dysfunction ${ }^{1,2}$.

\footnotetext{
Correspondence: Hwajin Kim (hwajin1@gmail.com) or Sang Won Park (parksw@gnu.ac.kr)

'Department of Pharmacology, Institute of Health Sciences, Gyeongsang National University School of Medicine, Jinju 52727, Republic of Korea

${ }^{2}$ Department of Convergence Medical Sciences, Institute of Health Sciences, Gyeongsang National University Graduate School, Jinju 52727, Republic of Korea
}

Hepatic IR aggravates hepatic tissue damage through ATP depletion, production of reactive oxygen species (ROS), and inflammatory responses, which cause necrotic and apoptotic cell death ${ }^{3}$. IR injury affects parenchymal hepatocytes, nonparenchymal cells (liver sinusoidal endothelial, Kupffer, and hepatic stellate cells), and extrahepatic components (cytokines) ${ }^{4}$. Unfortunately, effective therapeutic strategies for treating or preventing this devastating syndrome are clinically limited, despite our advanced understanding of IR injury mechanisms.

Autophagy is a highly conservative cellular process that degrades and recycles misfolded or dysfunctional proteins and damaged organelles to maintain cellular homeostasis ${ }^{5}$. 
However, in cases of alcoholic liver, non-alcoholic fatty liver, viral hepatitis, toxin-induced hepatitis, and hepatocellular carcinoma, autophagy is impaired ${ }^{6}$. Autophagy induction ameliorates hepatic IR dysfunction by activating the heme oxygenase-1 (HO-1) pathway ${ }^{7,8}$.

Mitochondria are major organelles responsible for ATP generation and cellular homeostasis. During the ischemic period, ATP is depleted due to a lack of oxygen, resulting in a switch to anaerobic respiration. After reperfusion, mitochondrial function is disrupted, leading to excessive ROS generation, the opening of mitochondrial permeability transition pores, inflammation, and subsequent cell death ${ }^{3,9}$. Hepatic IR injury is attenuated by inhibition of oxidative stress and mitochondrial respiratory dysfunc$\operatorname{tion}^{10}$, as well as stimulation to restore mitochondrial mass and membrane potential ${ }^{10,11}$. Here, we investigated the effect of nobiletin on autophagy and mitochondrial regulation during hepatic IR.

Sirtuin-1 (SIRT-1) is an important metabolic sensor that responds to cellular stress, starvation, and caloric restriction $^{12}$. Activation of SIRT-1 promotes the transcription of genes that regulate mitochondrial biogenesis to maintain energy and metabolic homeostasis ${ }^{13,14}$. Indeed, SIRT-1 is the principal target of many phytoflavonoids, including resveratrol, which has beneficial metabolic and antiaging effects ${ }^{15}$. SIRT-1 activation by such phytochemicals regulates peroxisome proliferatoractivated receptor gamma coactivator-1 alpha (PGC-1 $\alpha)$ mediated mitochondrial biogenesis and mitophagy. In addition, SIRT-1 deacetylates members of the forkhead box $\mathrm{O}$ (FoxO) family and affects downstream pathways controlling autophagy ${ }^{16,17}$. Pharmacological stimulation of SIRT-1 attenuates hepatic IR injury through mitochondrial recovery and enhanced autophagy ${ }^{11}$. Thus, we aimed to determine whether nobiletin protects against hepatic IR through SIRT-1.

Nobiletin (Fig. 1) is a polymethoxyflavone primarily present in citrus fruits and has a broad range of beneficial properties, including antioxidant, anti-inflammatory ${ }^{18,19}$, anti-cancer ${ }^{20}$, and antidiabetic ${ }^{21}$ activities. Nobiletin reduces cerebral ischemic injury by upregulating nuclear factor (erythroid-derived 2)-like-2 (Nrf2) and HO-1, attenuates IR injury following liver transplantation through the suppression of Kupffer cell activation, and

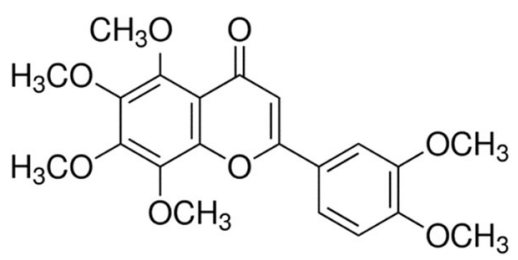

Fig. 1 Chemical structure of nobiletin protects against acute myocardial infarction by restoring impaired autophagic flux ${ }^{19,22,23}$. However, the detailed molecular mechanisms by which nobiletin regulates autophagy and mitochondrial function in hepatic IR injury remain undefined. Thus, we investigated the effect of nobiletin on hepatic IR injury and its underlying molecular mechanisms.

\section{Materials and methods \\ Experimental animals}

Male C57BL/6 mice (9-week-old) were purchased from Koatech (Pyeongtaek, Korea) and maintained in the animal facility at Gyeongsang National University (GNU). All animal experiments were approved by the Institutional Board of Animal Research at GNU and performed in accordance with the National Institutes of Health guidelines for laboratory animal care. Mice were housed under alternating 12 -h light/dark cycles with a relative humidity of $60 \pm 10 \%$ and a controlled temperature of $22 \pm 2^{\circ} \mathrm{C}$. Animals were provided with standard chow ad libitum and tap water.

\section{Animal model of hepatic IR}

Mice were randomly divided into five groups of six mice each: sham, hepatic IR (HIR) treated with the vehicle or nobiletin, and HIR pretreated with a SIRT-1 inhibitor and treated with the vehicle or nobiletin. The SIRT-1 inhibitor, EX-527, was administered intraperitoneally (i.p.) once daily for 3 days prior to ischemia. Anesthetized mice were placed supine on a heating pad under a heat lamp to maintain body temperature throughout the surgical procedures. After a midline abdominal incision, the liver hilum was exposed, and the hepatic artery and portal vein were identified. A microaneurysm clip was placed across the hilum of the left and median lobes to produce a $70 \%$ hepatic warm ischemia. The right lobes remained perfused to prevent intestinal blood congestion. After $60 \mathrm{~min}$ of ischemia, the clip was removed, and the abdomen was closed with sutures. Nobiletin $(5 \mathrm{mg} / \mathrm{kg})$ or the vehicle (0.025\% carboxymethyl cellulose) was i.p. injected at the start of reperfusion. The nobiletin dose was determined based on a pilot study (Supplementary Fig. 1). Blood and ischemic hepatic tissues were collected at $5 \mathrm{~h}$ of reperfusion. The median and left lobes were immediately removed and either fixed in $10 \%$ formalin or snap-frozen in liquid nitrogen and stored at $-80^{\circ} \mathrm{C}$.

\section{Biochemical assays}

Plasma alanine aminotransferase (ALT) and aspartate aminotransferase (AST) were measured using a commercial assay kit (IVD Lab Co., Ltd., Uiwang, Korea) and a spectrophotometer (Shimadzu UV-1800 spectrophotometer, Tokyo, Japan). Hepatic glutathione (GSH) levels were assayed from liver tissue lysates using a GSH/ 
glutathione disulfide (GSSG) Ratio Detection Kit II (Fluorometric-Green, Abcam 205811; Cambridge, UK) according to the manufacturer's instructions, and the wavelength was determined $(\mathrm{Ex} / \mathrm{Em}=490 / 520 \mathrm{~nm})$ using a Tecan Infinite M200 PRO Microplate Reader (Tecan Austria GmbH, Grödig, Austria).

\section{Histological evaluation of liver injury}

The fixed liver tissues were embedded in paraffin, and the blocks were cut into $5-\mu \mathrm{m}$ sections. The sections were then stained with hematoxylin and eosin (H\&E) according to standard procedures and examined using a Nikon Eclipse Ti-U microscope (Tokyo, Japan). The histological injury score for each sample was expressed as the sum of the individual scores for three different parameters based on the following Suzuki criteria: congestion (None $=0$, Minimal $=1, \quad$ Mild $=2, \quad$ Moderate $=3, \quad$ Severe $=4)$, vacuolization (None $=0$, Minimal $=1$, Mild $=2$, Moderate $=3$, Severe $=4$ ), and necrosis (None $=0$, Single Cell Necrosis $=1,<30 \%=2, \quad 30-60 \%=3,>60 \%=4)$; scores for each parameter ranged from 0 to 4 , with a maximum score of $12^{24}$.

\section{Terminal deoxynucleotidyl transferase dUTP nick-end labeling assay}

Terminal deoxynucleotidyl transferase dUTP nick-end labeling (TUNEL) staining was performed to evaluate the degree of apoptosis using an In Situ Cell Death Fluorescein Detection Kit (Roche Molecular Biochemicals, Mannheim, Germany) according to the manufacturer's instructions. The images were captured using a Nikon Eclipse Ti-U microscope (Tokyo, Japan).

\section{Western blot analysis}

Liver tissues were homogenized in ice-cold radioimmunoprecipitation assay (RIPA) buffer with protease inhibitors (Thermo Fisher Scientific, Waltham, MA, USA), sonicated, and incubated for $30 \mathrm{~min}$ on ice. After centrifugation, the supernatant was transferred to a clean tube, and the protein concentration was determined using a Pierce ${ }^{\mathrm{TM}}$ BCA Protein Assay Kit (Thermo Fisher Scientific). The protein lysates were separated using SDSPAGE and transferred to PVDF membranes. After blocking with $5 \%$ skim milk, the membranes were incubated with primary antibodies against SIRT-1, FOXO3a, phosphorylated AMP-activated protein kinase (p-AMPK), AMPK, p-AKT, AKT, uncleaved caspase-3, cleaved caspase-3, uncleaved poly (ADP-ribose) polymerase 1 (PARP-1), cleaved PARP-1, autophagy-related gene5 (ATG5), ATG7, ATG12, light-chain 3B (LC3B), and P62 (Cell Signaling Technology, Danvers, MA, USA); dynamin-related protein 1 (DRP-1; Santa Cruz Biotechnology, Dallas, TX, USA); PGC-1 $\alpha$, p-FOXO3a, mitofusin 2 (MFN-2), 4-hydroxynonenal (4-HNE), matrix metalloproteinase 2 (MMP-2), MMP-9, and p-84 (Abcam); optic atrophy 1 (OPA-1; BD Biosciences, Franklin Lakes, NJ, USA); and $\beta$-actin (Sigma, St. Louis, MO, USA) in blocking solution at $4{ }^{\circ} \mathrm{C}$ overnight. The membranes were then incubated with the appropriate horseradish peroxidase (HRP)-conjugated secondary antibodies (Bio-Rad, Hercules, CA, USA) at room temperature for $1 \mathrm{~h}$ and then visualized with the ECL substrate (Bio-Rad). The ChemiDoc XRS + System (Bio-Rad) was used to evaluate the density of the protein bands. The relative protein expression was quantified using Image $\mathrm{Lab}^{\mathrm{TM}}$ software (Bio-Rad).

\section{Nuclear-cytoplasmic fractionation}

Nuclear-cytoplasmic fractionation was conducted using NE-PER ${ }^{\mathrm{TM}}$ nuclear and cytoplasmic extraction reagents (Life Technologies, 78855, Carlsbad, CA, USA) according to the manufacturer's instructions. The protein concentration was determined using a Pierce ${ }^{\mathrm{TM}}$ BCA Protein Assay Kit (Thermo Fisher Scientific), and equal amounts of proteins were separated by SDS-PAGE. To assess fractionation efficiency, cytoplasmic and nuclear fractions were confirmed by immunoblotting with anti- $\beta$-tubulin (Sigma T8328) and anti-P84 (Abcam 46545), respectively.

\section{Quantitative real-time PCR (qRT-PCR) analysis}

The total RNA was extracted with TRIzol (Invitrogen, Carlsbad, CA, USA) and converted into cDNA using the RevertAid Reverse Transcription System (Thermo Fisher Scientific), according to the manufacturer's protocol. qPCR was performed with a CFX Connect Real-Time PCR System using iQ SYBR Green Supermix (Bio-Rad). Relative mRNA levels were normalized to those of GAPDH. The primer sequences are listed in Table 1.

\section{Statistical analysis}

Statistical differences among the groups were determined using one-way analysis of variance followed by Bonferroni post hoc analysis. The values were expressed as the mean \pm SEM. A $p$-value $<0.05$ was considered statistically significant.

\section{Results}

Nobiletin attenuated hepatocellular damage induced by hepatic IR injury

Liver damage was assessed by measuring plasma ALT and AST, which were significantly increased in the hepatic IR group compared with the sham group (Fig. 2a). In contrast, nobiletin treatment at the start of reperfusion significantly decreased ALT and AST levels by $53.9 \%$ and $64.3 \%$, respectively, compared with the hepatic IR group (Fig. 2a). These results were consistent with histopathological changes in liver tissues. Liver tissues from the hepatic IR group demonstrated increased necrotic area, sinusoidal 
Table 1 The primer sequences used for qRT-PCR analysis in this study

\begin{tabular}{|c|c|}
\hline Gene & Primers $\left(5^{\prime}-3^{\prime}\right)$ \\
\hline \multirow[t]{2}{*}{ ATG5 } & Forward: ACCAAATCGTTACATATTCC \\
\hline & Reverse: CAAGGGTTCTTCTAAACTTG \\
\hline \multirow[t]{2}{*}{ ATG7 } & Forward: CGCTTGACGTTGGAGTTCAGTG \\
\hline & Reverse: GTGTTGTGCAGGGTTCCCATG \\
\hline \multirow[t]{2}{*}{ ATG12 } & Forward: AGCTCTTCAGTCCTGTCATTTC \\
\hline & Reverse: ACTCCTGGTTCACTCTTCCT \\
\hline \multirow[t]{2}{*}{ Beclin-1 } & Forward: GTCTAAGGCGTCCAGCAGCAC \\
\hline & Reverse: TGGGCTGTGGTAAGTAATGGAGC \\
\hline \multirow[t]{2}{*}{ DRP-1 } & Forward: ACCAAAGTACCTGTAGGCGATC \\
\hline & Reverse: CATGGCATCAGTACCCGCAT \\
\hline \multirow[t]{2}{*}{ FOXO3a } & Forward: CTGTCCTATGCCGACCTGATCAC \\
\hline & Reverse: CATTCTGAACGCGCATGAAGCG \\
\hline \multirow[t]{2}{*}{$\mid L-1 \beta$} & Forward: TTTGTACAAGGAGAACCAAG \\
\hline & Reverse: TTTCATTACACAGGACAGGT \\
\hline \multirow[t]{2}{*}{ IL-6 } & Forward: CCAATTCATCTTGAAATCAC \\
\hline & Reverse: GGAATGTCCACAAACTGATA \\
\hline \multirow[t]{2}{*}{ MFN-2 } & Forward: ACCGTCAAGAAGGATAAGCGACAC \\
\hline & Reverse: GTGTTCCTGTGGGTGTCTTCAAGG \\
\hline \multirow[t]{2}{*}{ MMP-2 } & Forward: GTTCAACGGTCGGGAATACA \\
\hline & Reverse: GCCATACTTGCCATCCTTCT \\
\hline \multirow[t]{2}{*}{ MMP-9 } & Forward: CTGGAACTCACACGACATCTT \\
\hline & Reverse: TCCACCTTGTTCACCTCATTT \\
\hline \multirow[t]{2}{*}{ NRF1 } & Forward: GAGCACGGAGTGACCCAAAC \\
\hline & Reverse: TGTACGTGGCTACATGGACCT \\
\hline \multirow[t]{2}{*}{ OPA-1 } & Forward: CCAAGAACGAGTTGGAGAAGATGC \\
\hline & Reverse: CACGTCATTGCATTCCAGCTCAGA \\
\hline \multirow[t]{2}{*}{ PGC-1a } & Forward: AGCCGTGACCACTGACAACGAG \\
\hline & Reverse: GCTGCATGGTTCTGAGTGCTAAG \\
\hline \multirow[t]{2}{*}{ SIRT-1 } & Forward: ACCAAATCGTTACATATTCC \\
\hline & Reverse: CAAGGGTTCTTCTAAACTTG \\
\hline \multirow[t]{2}{*}{ TFAM } & Forward: CCAAGTCAGCTGATGGGTATGG \\
\hline & Reverse: CCTGAGCCGAATCATCCTTTGC \\
\hline \multirow[t]{2}{*}{ TNFa } & Forward: GAGCAATGACTCCAAAGTAG \\
\hline & Reverse: CCAATTCATCTTGAAATCAC \\
\hline
\end{tabular}

congestion, and cytoplasmic vacuolization of hepatocytes compared with the liver tissues of the sham group. These features were significantly attenuated by nobiletin treatment (Fig. 2b). The extent of liver damage was quantitatively presented based on the Suzuki classification (Fig. 2c).
To investigate the effect of nobiletin on hepatic apoptosis induced by IR injury, we performed a western blot analysis to assess cleavage of caspase- 3 and PARP-1. Cleavage of caspase-3 and PARP-1 significantly increased in the hepatic IR group compared with the sham group, which was attenuated by nobiletin treatment (Fig. 2d). TUNEL staining showed a significant increase in the number of TUNEL-positive cells after IR injury, which was decreased upon nobiletin treatment (Fig. 2e).

\section{Nobiletin decreased oxidative stress and induction of inflammatory cytokines and MMPs induced by hepatic IR injury \\ Oxidative stress was then assessed by 4-HNE, a marker} of lipid peroxidation. The levels of 4-HNE increased significantly in the hepatic IR group compared with the sham group, which decreased upon nobiletin treatment (Fig. 3a). Hepatic levels of the total and reduced GSH and GSH/GSSG ratios were assessed as indicators of endogenous antioxidant system activity. We found that the total GSH decreased after IR, but there was no significant increase upon nobiletin treatment. However, the reduced GSH and GSH/GSSG ratio markedly increased upon nobiletin treatment compared with the hepatic IR group (Fig. 3b).

We next investigated whether nobiletin reduces inflammatory cytokine and MMP expression during hepatic IR using real-time PCR analysis. Hepatic IRinduced mRNA expression of the proinflammatory cytokines tumor necrosis factor- $\alpha$ (TNF- $\alpha)$, interleukin- $1 \beta$ (IL-1 $\beta$ ), and interleukin-6 (IL-6) was significantly reduced upon nobiletin treatment (Fig. 3c). Similarly, upregulation of MMP-9 and MMP-2 genes was reduced upon nobiletin treatment (Fig. 3d). Furthermore, western blot analysis demonstrated a significant increase in MMP-2 and MMP9 protein levels in the hepatic IR group, which was reduced by nobiletin treatment (Fig. 3e).

\section{Nobiletin attenuated hepatic IR injury through induction of autophagy}

We next investigated whether nobiletin induces autophagy in the liver following hepatic IR injury. The levels of autophagy-related proteins (ATG5, ATG12, and LC3B-II) decreased, and p62, a marker of autophagy deficit, significantly increased in the hepatic IR group compared with the sham group. In contrast, nobiletin treatment markedly increased the expression of autophagy-related proteins and decreased p62 protein accumulation in the liver following IR injury compared with the hepatic IR group (Fig. 4a). Similarly, nobiletin treatment significantly induced autophagy-regulatory genes (ATG5, ATG12, ATG7, and Beclin-1) during hepatic IR injury (Fig. 4b). 


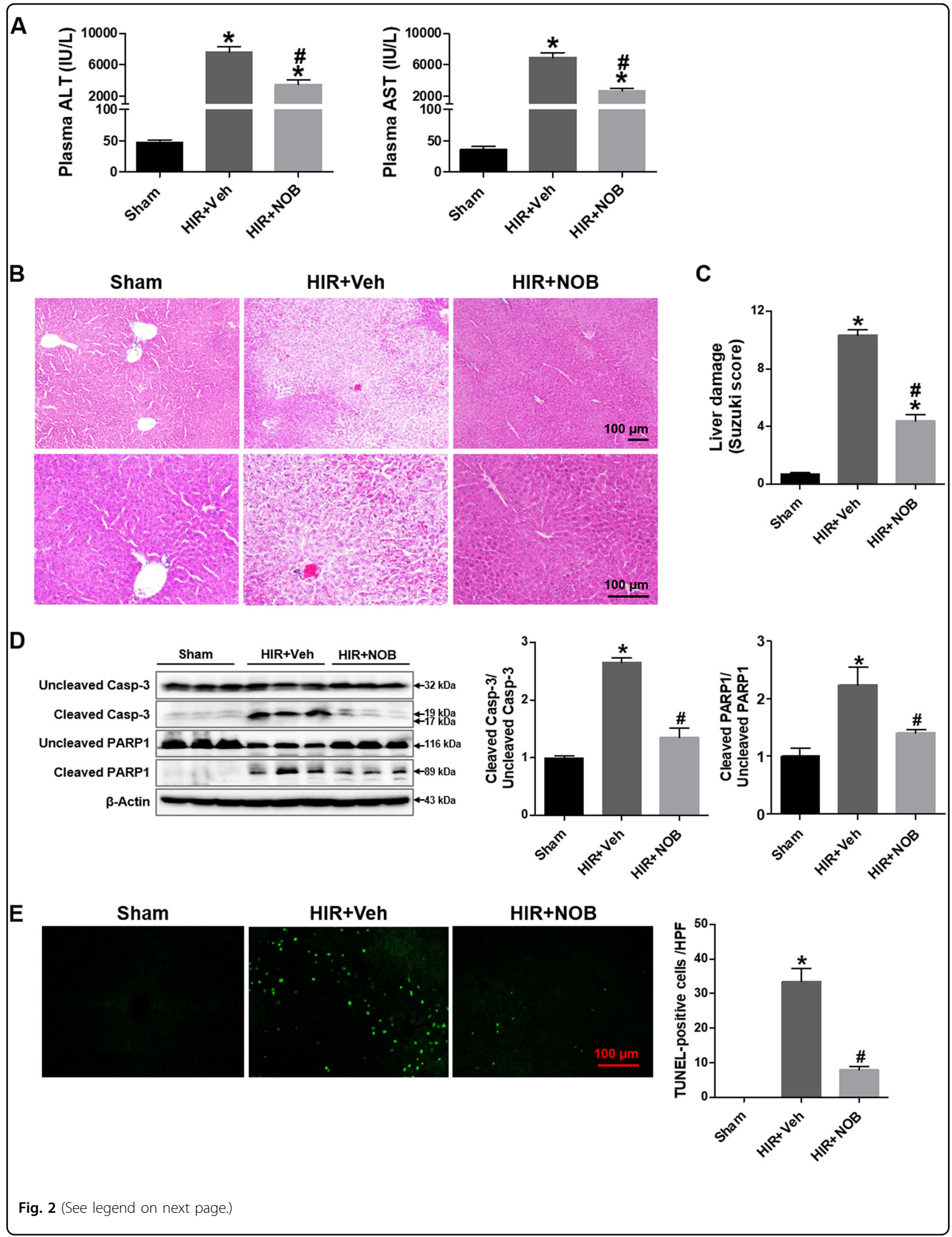


(see figure on previous page)

Fig. 2 Effect of nobiletin on hepatocellular damage during hepatic IR injury. Mouse livers were subjected to ischemia for 60 min followed by $5 \mathrm{~h}$ of reperfusion. The mice were treated with the vehicle or nobiletin $(5 \mathrm{mg} / \mathrm{kg})$ at the start of reperfusion. The liver tissues and blood were harvested from sham-operated (sham group) or hepatic IR-subjected (HIR group) mice. a Plasma ALT and AST levels were measured to assess the degree of liver injury. $\mathbf{b}$ Representative images of hematoxylin and eosin (H\&E) staining from liver sections to evaluate hepatic necrosis and pathological changes. c The extent of liver damage was graded by Suzuki score as described in the Materials and methods section. $\mathbf{d}$ The degree of hepatocellular apoptosis was determined by caspase- 3 and PARP-1 cleavage in liver tissue lysates using western blot analysis, and quantitative analysis is shown. e Apoptosis-positive cells were analyzed by TUNEL, and the numbers of apoptotic cells per $\times 200$ field image were counted. The data are presented as the mean \pm SEM $(n=6) .{ }^{*} p<0.05$ vs. Sham group, ${ }^{\#} p<0.05$ vs. HIR + Veh group. Scale bar, $100 \mu m$

Nobiletin protected against mitochondrial dysfunction by restoring mitochondrial biogenesis and fusion-fission dynamics in hepatic IR injury

Mitochondrial biogenesis is tightly regulated by PGC- $1 \alpha$ and the downstream nuclear respiratory factor 1 (NRF1) and mitochondrial transcription factor A (TFAM) pathways, which control mitochondrial turnover, content, and number to maintain diverse metabolic demands ${ }^{25,26}$. In this study, we found that mRNA expression of PGC- $1 \alpha$, NRF1, and TFAM was significantly increased upon nobiletin treatment in the hepatic IR group compared with the sham group (Fig. 5a). Moreover, mRNA expression of MFN-2 increased and DRP-1 expression decreased; however, OPA-1 expression was not significantly changed (Fig. 5a). We also found that protein expression of PGC-1 $\alpha, \mathrm{MFN}-2$, and OPA-1 was decreased, whereas DRP-1 expression was increased in the hepatic IR group, which was alleviated upon nobiletin treatment (Fig. 5b). These results indicate that nobiletin protects against IR-induced mitochondrial dysfunction by regulating $\mathrm{PGC}-1 \alpha$-mediated mitochondrial biogenesis and balancing fusion-fission dynamics.

\section{Nobiletin increased SIRT-1 and FOXO3a expression in hepatic IR injury}

Considering that SIRT-1/FOXO3a signaling plays a key role in autophagy induction ${ }^{27}$, we examined whether nobiletin affects SIRT-1 and FOXO3a expression in hepatic IR mice. We found that SIRT-1 and FOXO3a protein levels decreased in the hepatic IR group compared with the sham group, and nobiletin treatment significantly increased SIRT-1 and FOXO3a protein levels (Fig. 6a). Furthermore, qRT-PCR analysis revealed a significant increase in SIRT-1 and FOXO3a mRNA after nobiletin treatment in hepatic IR mice (Fig. 6b).

\section{Nobiletin decreased AKT phosphorylation to promote FOXO3a nuclear expression in hepatic IR injury}

FOXO3a plays a critical role in regulating autophagy, apoptosis, and oxidative stress in various disease conditions. In response to stress or pathological conditions, FOXO3a is phosphorylated by AKT and is retained in the cytosol, thereby inhibiting its transcriptional activity ${ }^{28,29}$.
Activated FOXO3a increases ATG gene transcription in mouse alcoholic livers ${ }^{30}$ and atrophying muscle cells ${ }^{31}$. We therefore investigated the mechanism of nobiletinmediated protection through the AKT and FOXO3a pathways (Fig. 7). The hepatic IR group exhibited a significant increase in AKT phosphorylation, which was reduced upon nobiletin treatment in whole-cell lysates. We then determined whether AKT phosphorylation levels were correlated with changes in the subcellular localization of FOXO3a. Analysis of cytosolic and nuclear fractions revealed that the nuclear versus cytosolic FOXO3a was significantly increased by its nuclear translocation from the cytosol following nobiletin treatment, which was coincident with a reduction in AKT phosphorylation and FOXO3a expression. These results suggest that nobiletin increases FOXO3a nuclear retention and subsequent transcriptional activity by inhibiting IR-induced AKT phosphorylation.

\section{SIRT-1 inhibition abolished the protective effect of nobiletin against hepatic IR injury}

A specific SIRT-1 inhibitor (EX-527) was used to investigate whether SIRT-1 activity is required for nobiletin-mediated protection against hepatic IR injury. We found that plasma ALT and AST were reduced in the nobiletin-treated hepatic IR group, and this effect was blocked by EX-527 treatment (Fig. 8a). H\&E staining further demonstrated that EX-527 treatment abolished the protective effect of nobiletin on reducing hepatic necrosis and corresponding Suzuki scores (Fig. 8b, c). Interestingly, EX-527-induced SIRT-1 inhibition often exacerbated IR-induced hepatic damage. These results indicate that SIRT-1 activity is required for a nobiletinmediated decrease in hepatocellular damage following hepatic IR injury.

SIRT-1 stimulation attenuates hepatic IR injury by activating PGC- $1 \alpha$ to promote mitochondrial biogenesis/ recovery and deacetylating FOXO3a to enhance autophagy $^{11,27,30}$. We demonstrated that nobiletin upregulated SIRT-1, with a simultaneous increase in FOXO3a expression. However, the effect was significantly blocked by SIRT-1 inhibition (Fig. 9a). FOXO3a-mediated induction of autophagy-related proteins (ATGs and LC3B-II) 


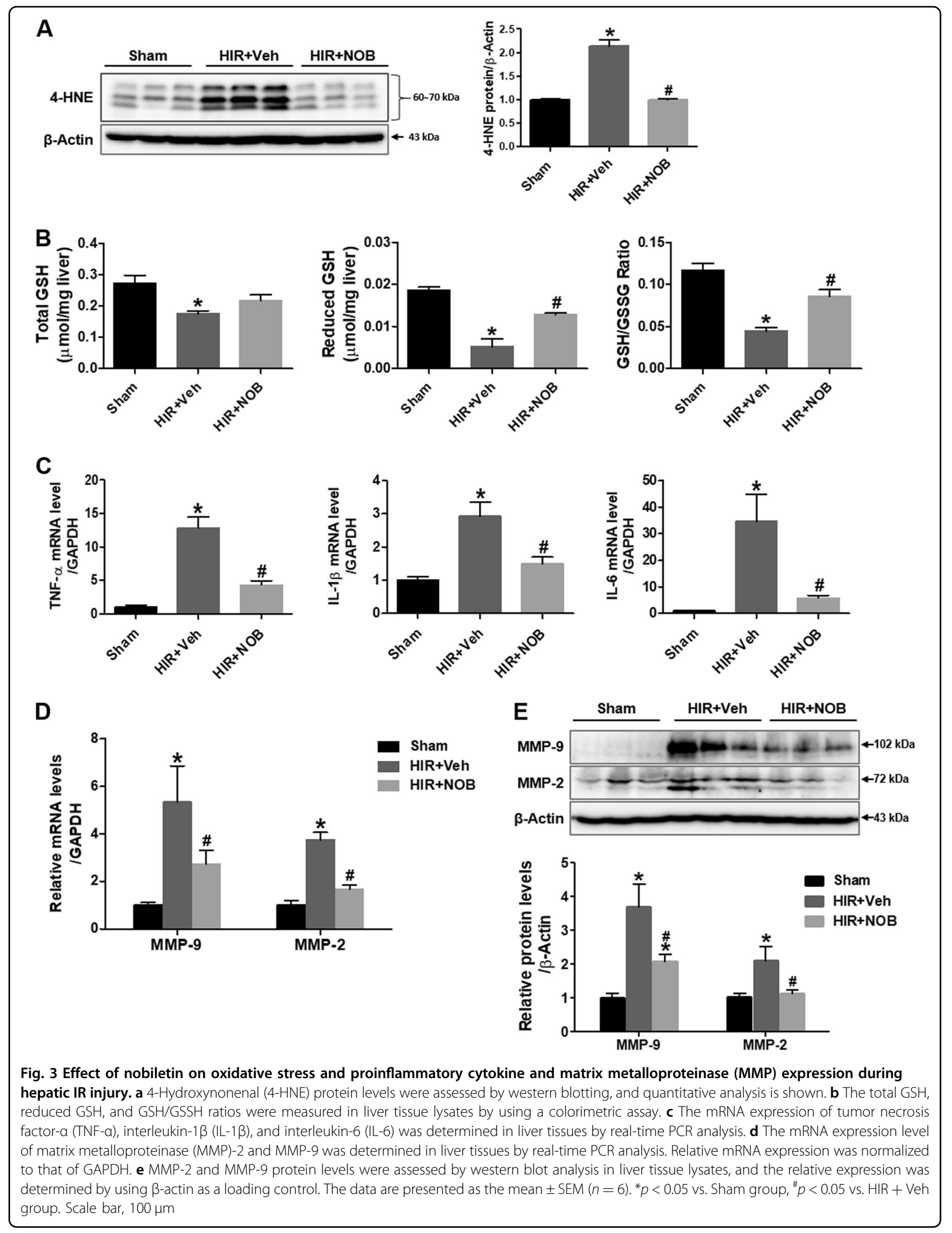



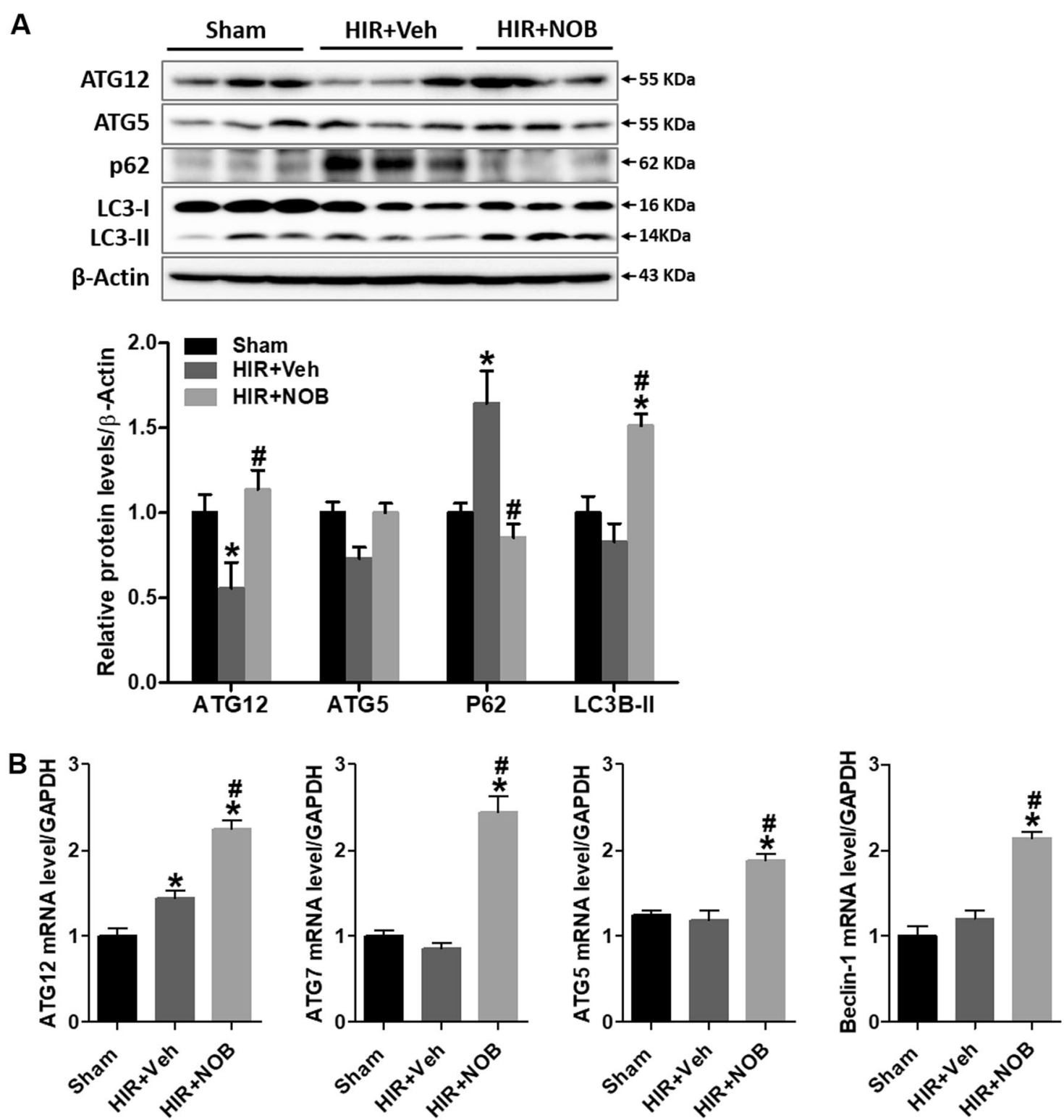

Fig. 4 Effect of nobiletin on autophagy response during hepatic IR injury. a The autophagy-related proteins ATG5, ATG12, LC3B, and p62 were examined by western blot analysis in liver tissue lysates, and relative protein expression was determined by using $\beta$-actin as a loading control. $\mathbf{b}$ The mRNA expression of ATG5, ATG7, ATG12, and Beclin-1 was determined in liver tissues by real-time PCR analysis. Relative mRNA expression was normalized to that of GAPDH. The data are presented as the mean \pm SEM $(n=6) .{ }^{*} p<0.05$ vs. Sham group, ${ }^{\#} p<0.05$ vs. HIR + Veh group

was attenuated by EX-527 treatment, with significant accumulation of p62 (Fig. 9b). PGC-1 $\alpha$ and mitochondrial fusion versus fission was upregulated by nobiletin, but the effect was abolished by EX-527. These results indicate that SIRT-1 activity is specifically required for FOXO3amediated induction of autophagy, PGC- $1 \alpha$-mediated mitochondrial biogenesis, and balancing mitochondrial fusion-fission dynamics, resulting in alleviation of hepatic IR injury (Fig. 9c). A summarized molecular mechanism of the nobiletin effect is depicted in Supplementary Fig. 2, illustrating that SIRT-1 is an essential upstream regulator of PGC-1 $\alpha$ and FOXO3a, enhancing mitochondrial and autophagy function and protecting the liver against hepatic IR injury.

\section{Discussion}

We demonstrated that nobiletin attenuates hepatic IR injury in the following ways: (1) nobiletin enhances autophagy and mitochondrial biogenesis and dynamics, (2) nobiletin induces autophagy through a SIRT-1/ FOXO3a signaling pathway, (3) nobiletin-mediated control of mitochondrial biogenesis depends on a PGC- $1 \alpha /$ 

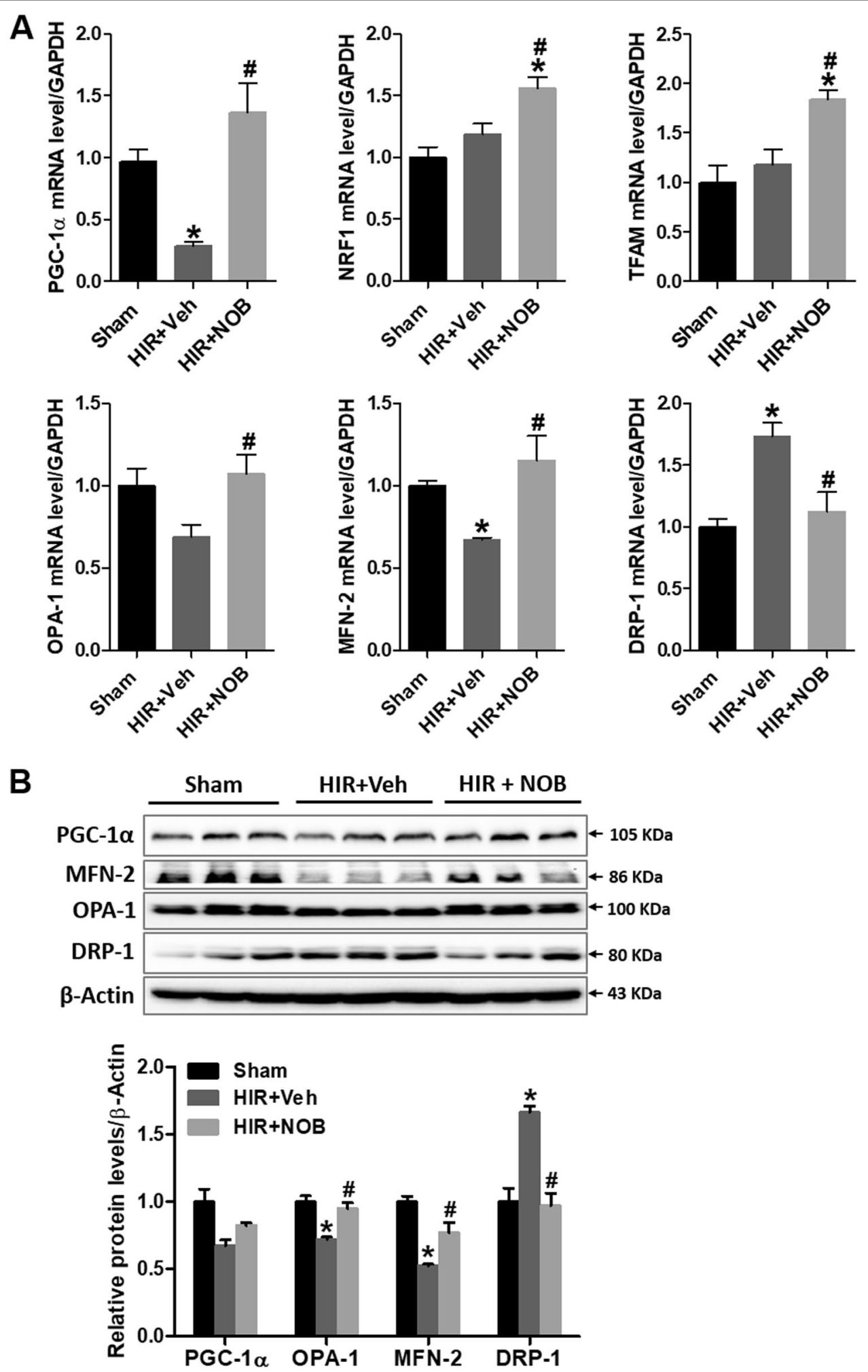

Fig. 5 Effect of nobiletin on mitochondrial biogenesis and fusion-fission dynamics during hepatic IR injury. a The mRNA expression of PGC1a, NRF1, TFAM, MFN-2, OPA-1, and DRP-1 was determined in liver tissues by real-time PCR analysis. Relative mRNA expression was normalized to that of GAPDH. $\mathbf{b}$ The protein expression level of PGC-1a, MFN-2, OPA-1, and DRP-1 was assessed in liver tissue lysates by western blotting, and the relative expression was determined by using $\beta$-actin as a loading control. Data are presented as the mean $\pm \operatorname{SEM}(n=6)$. ${ }^{*} p<0.05$ vs. Sham group, ${ }^{\#} p$ $<0.05$ vs. HIR + Veh group 


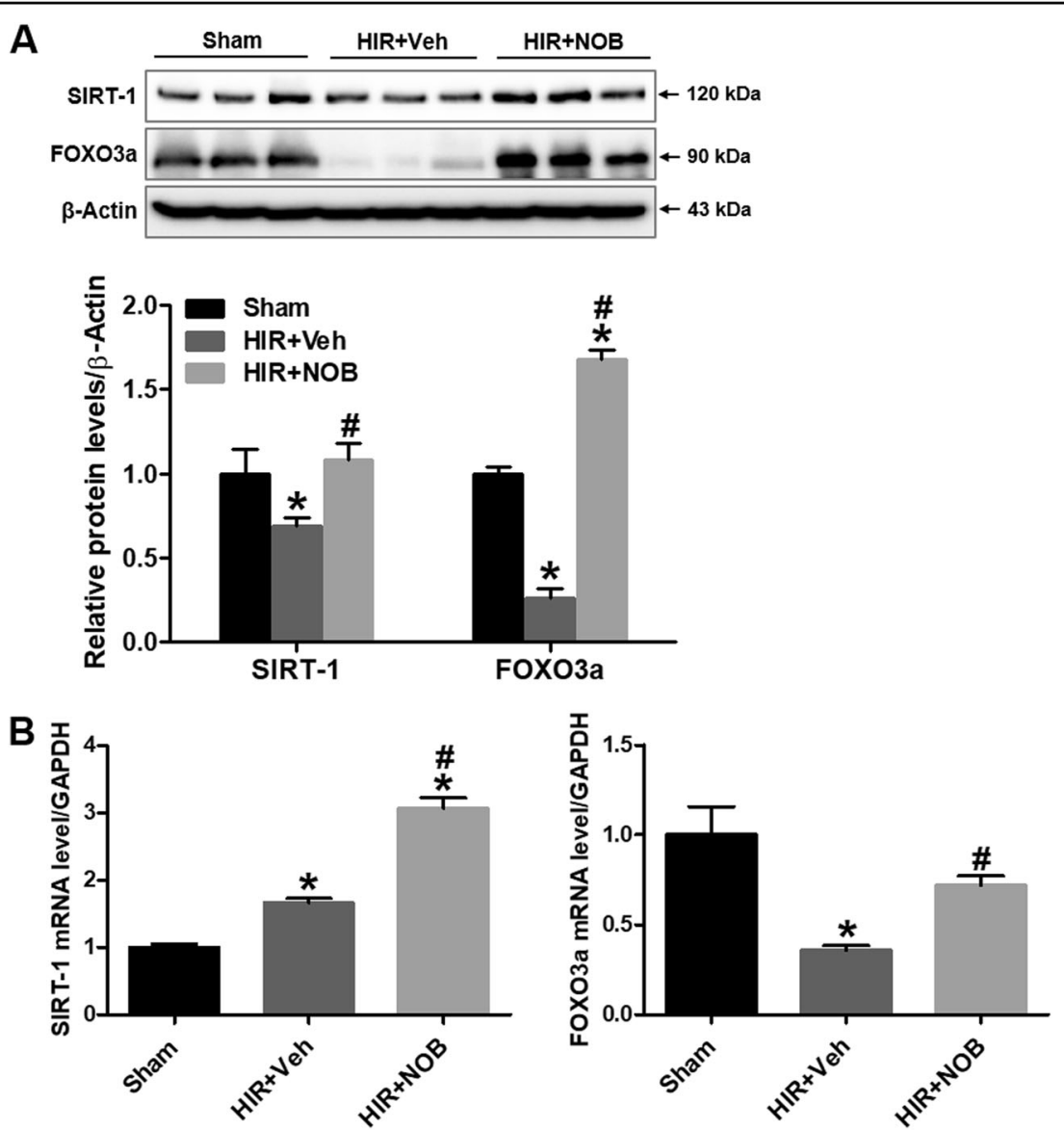

Fig. 6 Effect of nobiletin on SIRT-1 and FOXO3a expression during hepatic IR injury. a SIRT-1 and FOXO3a protein expression was examined by western blot analysis in the liver tissue lysates, and relative expression was determined by using $\beta$-actin as a loading control. b The mRNA expression of SIRT-1 and FOXO3a was determined in liver tissue lysates by real-time PCR analysis. Relative mRNA expression was normalized to that of GAPDH. The data are presented as the mean \pm SEM $(n=6) .{ }^{*} p<0.05$ vs. Sham group, ${ }^{*} p<0.05$ vs. HIR + Veh group

NRF1/TFAM signaling pathway, (4) nobiletin improves mitochondrial dynamics by regulating the expression of fusion and fission proteins, and (5) nobiletin reduces oxidative damage, the expression of MMPs and inflammation.

Hepatic IR injury is the primary cause of acute or chronic liver damage after liver transplantation or dissection, and is characterized by oxidative stress, inflammation, disruption of epithelial integrity, and impaired autophagy and mitochondrial function. These changes lead to hepatocyte necrosis and apoptosis, which contribute to organ failure ${ }^{32}$. We propose that enhancing autophagy and mitochondrial function is a promising therapeutic strategy to attenuate IR-induced damage.

Nobiletin, chemically 5,6,7,8,3',4'-hexamethoxyflavone, is a flavonoid abundant in citrus peel that has demonstrated various pharmacological activities against cardiovascular and metabolic disorders, inflammation, and cancers $^{33}$. The biological activities of nobiletin depend on its chemical structure; nobiletin is absorbed easily due to its lipophilic nature and high permeability. An X-ray crystal structure study revealed that the chromene and arene rings and the bound methoxy groups create a chiral molecule that can adopt a variety of conformations ${ }^{34}$. This molecular flexibility may support a wide range of physiological activities by binding the different target receptors of specific signaling pathways.

Since nobiletin is known as an MMP inhibitor, we first determined the effect of nobiletin on MMP-2 and -9 expression during hepatic IR injury. MMPs, proteolytic enzymes that degrade the extracellular matrix proteins, have been linked to cancer and chronic inflammation ${ }^{35}$. MMP-9 is induced by leukocytes following hepatic IR and is important for leukocyte migration into inflamed livers and subsequent cytokine release ${ }^{36}$. MMP-2 plays an important role in hepatic vascular homeostasis, is expressed in human fibrotic livers, and can either promote or inhibit inflammation in a condition-dependent manner ${ }^{37}$. Interestingly, an MMP-2 deletion enhances MMP-9 activity and exacerbates hepatic IR injury ${ }^{38}$, while 

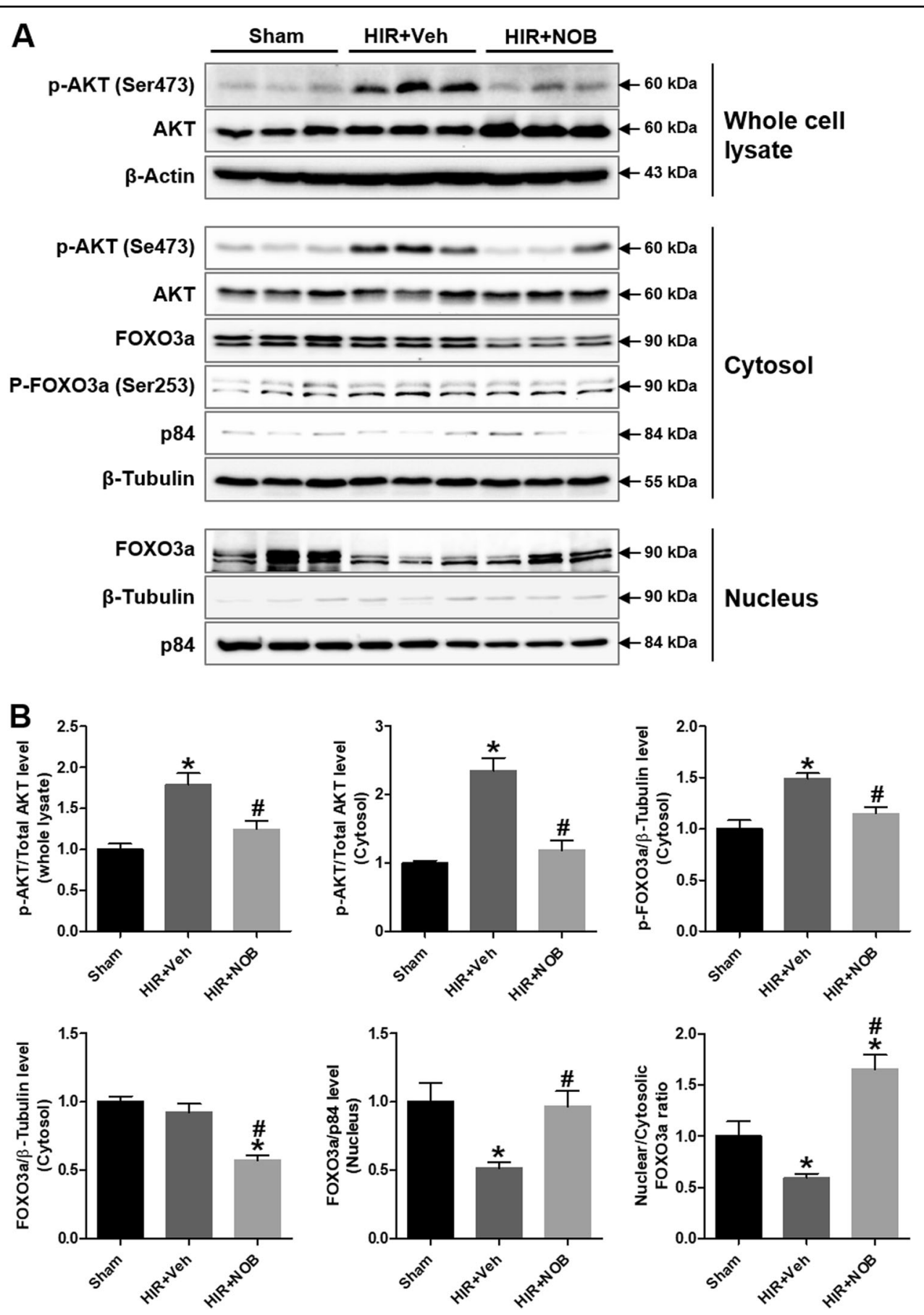

Fig. 7 Effect of nobiletin on AKT phosphorylation and FOXO3a expression during hepatic IR injury. Whole-liver tissue lysates and the nuclear and cytosolic fractions were prepared to evaluate AKT phosphorylation and FOXO3a localization. a The protein expression of AKT, p-AKT (Ser473), FOXO3a, and p-FOXO3a (Ser253) was examined by western blot analysis. b The relative expression was determined by using total AKT, $\beta$-tubulin (cytosolic marker), and p84 (nuclear marker) as loading controls. The data are presented as the mean \pm SEM $(n=6)$. ${ }^{*} p<0.05$ vs. Sham group, ${ }^{*} p<$ 0.05 vs. HIR + Veh group

MMP-9 deletion protects against hepatic IR injury in mice $^{39}$. Treatment with bortezomib, a proteasome inhibitor, protects against steatotic liver IR injury by inhibiting MMP-2 and MMP-9 activation and reducing proinflammatory cytokines $^{40}$. Similarly, our results showed that nobiletin inhibited expression of both MMP2 and MMP-9 and downregulated proinflammatory cytokines following hepatic IR. The molecular mechanism 

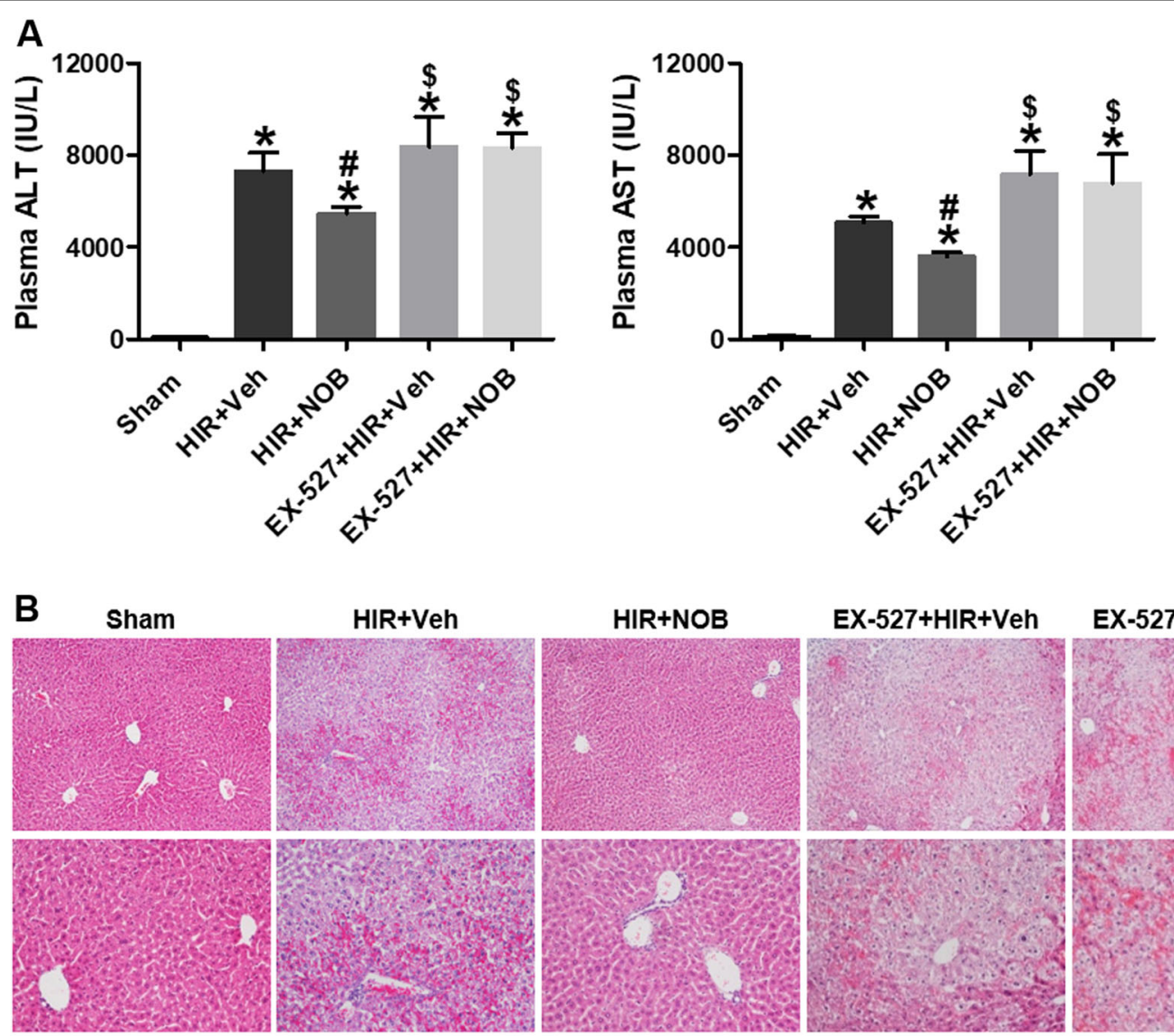

EX-527+HIR+NOB
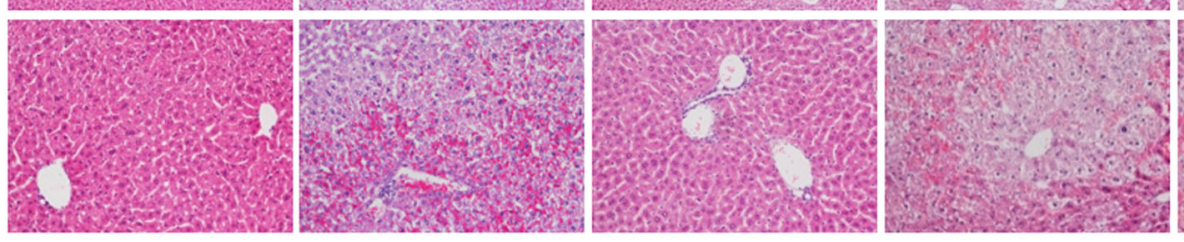

$100 \mu \mathrm{m}$

C

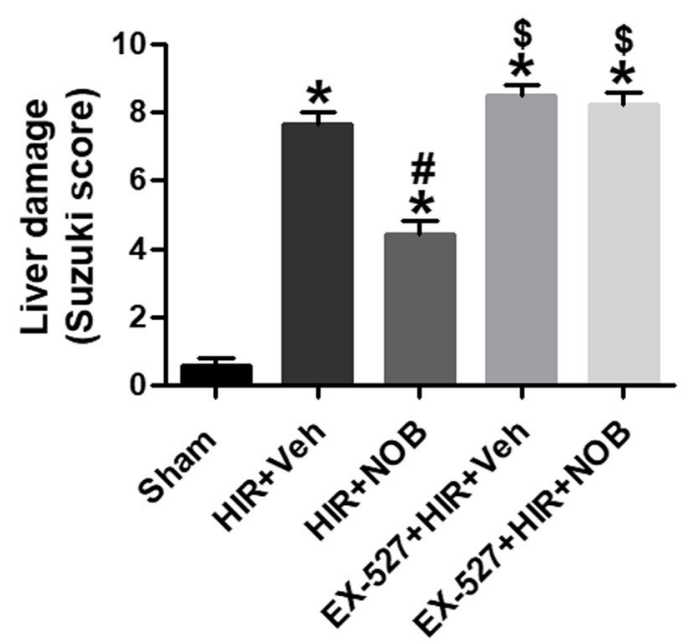

Fig. 8 Effect of SIRT-1 inhibition on nobiletin-mediated protection against hepatocellular damage during hepatic IR injury. Mouse livers were subjected to ischemia for $60 \mathrm{~min}$ followed by $5 \mathrm{~h}$ of reperfusion. The mice were treated with vehicle or SIRT-1 inhibitor EX527 (5 mg/kg) for 3 days before ischemia and then treated with nobiletin $(5 \mathrm{mg} / \mathrm{kg})$ at the start of reperfusion. The liver tissues and blood were harvested after reperfusion. Plasma ALT and AST levels (a), representative images of H\&E staining (b), and liver damage based on the Suzuki scoring system (c) are shown. The data are presented as the mean \pm SEM $(n=6) .{ }^{*} p<0.05$ vs. Sham group, ${ }^{\#} p<0.05$ vs. HIR + Veh group, ${ }^{\$} p<0.05$ vs. HIR + NOB group. Scale bar, $100 \mu \mathrm{m}$ 


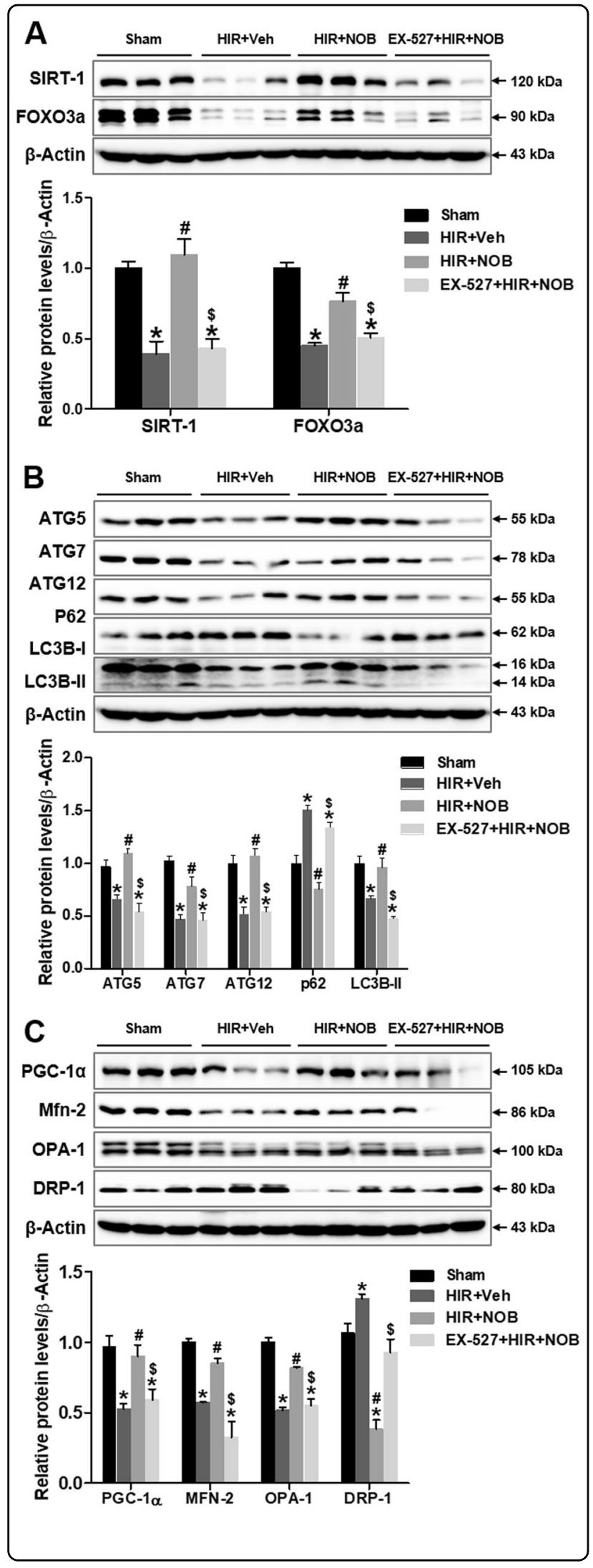

Fig. 9 Effect of SIRT-1 inhibition on nobiletin-mediated autophagy induction and mitochondrial biogenesis and dynamics during hepatic IR injury. Whole-liver tissue lysates were prepared to examine protein expression levels by western blot analysis; the blots of SIRT-1, FOXO3a (a), autophagy-related proteins ATG5, ATG7, ATG12, LC3B, p62 (b), and mitochondria-regulating proteins PGC-1a, MFN-2, OPA-1, DRP-1 (c) are shown. The relative expression was determined by using $\beta$-actin as a loading control. The data are presented as the mean \pm SEM $(n=6)$. ${ }^{*} p<0.05$ vs. Sham group, $" p<0.05$ vs. HIR + Veh group, ${ }^{\$} p<0.05$ vs. HIR + NOB group

of nobiletin-mediated regulation of MMPs requires further study.

Mitochondrial dysfunction is a central feature of IR injury, as mitochondria are important for energy homeostasis and directly mediate oxidative stress and cell death ${ }^{41}$. Mitochondria play a key role in initiating apoptosis through a permeability transition followed by excessive ATP depletion and ROS production, and mitochondrial breakdown leads to necrosis and apopto$\mathrm{sis}^{42}$. In this study, nobiletin attenuated oxidative stress and hepatic cell death induced by necrosis and apoptosis during hepatic IR injury.

Mitochondrial biogenesis is a critical control mechanism for mitochondrial turnover, content, and number, all of which are needed to meet metabolic energy demands and to prevent cell death in various disease ${ }^{25}$. PGC- $1 \alpha$ is a major regulator of mitochondrial biogenesis that activates different transcription factors, including NRF1 and TFAM. NRF1 is a transcription factor that stimulates mitochondrial enzyme expression and directly interacts with TFAM to promote transcription and replication of mitochondrial DNA (mtDNA) ${ }^{43}$. Therefore, we examined PGC-1 $\alpha$, NRF1, and TFAM expression, which positively regulates mitochondrial biogenesis. Recent studies found that cilostazol or genipin treatment stimulates mitochondrial biogenesis via induction of the PGC- $1 \alpha / \mathrm{NRF} 1 /$ TFAM pathway, leading to improved mitochondrial functioning during hepatic IR injury ${ }^{26,44}$. Similarly, in this study, hepatic IR decreased PGC- $1 \alpha$, NRF1, and TFAM expression, but these factors were significantly upregulated upon nobiletin treatment. Nobiletin has the potential to enhance mitochondrial biogenesis and protect against IR-induced mitochondrial dysfunction.

SIRT-1 plays an important role in many physiological processes of cellular energetics, metabolism, and aging, in particular by interacting with PGC- $1 \alpha^{13,45}$, SIRT-1 interacts with and deacetylates PGC- $1 \alpha$ to increase its activity and control mitochondrial function. SIRT-1 activation reduces IR-induced oxidative damage by enhancing mitochondrial biogenesis and restoring impaired mitophagy and mitochondrial dynamics ${ }^{26}$. Similarly, our 
results showed that nobiletin increased SIRT-1 expression and upregulated PGC- $1 \alpha$ to promote mitochondrial biogenesis and dynamics, thus attenuating hepatic IR injury.

Mitochondrial dynamics play a critical role in determining mitochondrial morphology and function. Fusion-fission dynamics are regulated by the expression and activity of the fusion (MFN-1/2 and OPA-1) and fission (DRP-1) proteins ${ }^{46}$. DRP-1 inhibition protects against mitochondrial fragmentation and apoptosis in mice with senecionine-induced liver injury, while reduced endogenous MFN-2 expression impairs mitochondrial fusion, contributing to the pathogenesis of liver damage in patients with chronic liver cholestasis ${ }^{47,48}$. In addition, PGC- $1 \alpha$ stimulates MFN-2 mRNA and protein expression under altered energy-expenditure conditions, such as cold exposure, fasting, and IR, to control mitochondrial energy metabolism in the skeletal muscle, heart, and liver ${ }^{49-51}$. Our results show that nobiletin decreased DRP-1 and increased MFN-2 and OPA-1 expression to protect the mitochondria from excessive fragmentation and functional failure during hepatic IR injury.

Autophagy is activated when cells are under metabolic, ischemic, or hypoxic stress. Enhancing autophagy improves liver function by eliminating the abnormal or dysfunctional mitochondria ${ }^{52}$. Inhibiting autophagy increases mitochondrial oxidative stress and accelerates apoptotic and necrotic cell death during hepatic IR injury $^{53}$. Thus, autophagy induction could restore mitochondrial function after acute liver damage ${ }^{54}$. A previous study showed that nobiletin attenuates acute myocardial infarction by restoring impaired autophagic flux ${ }^{22}$. In this study, we revealed the molecular mechanism of autophagy induction by nobiletin during hepatic IR.

The FOXO family regulates autophagy in various organs, including the skeletal muscle, cardiomyocytes, and liver ${ }^{31,55}$. In addition, ethanol treatment increases the expression of autophagy-related genes through FOXO3a activation in mouse liver and primary hepatocytes ${ }^{56}$. FOXO3a activity is regulated by posttranslational modifications, including phosphorylation, acetylation, and ubiquitination ${ }^{16}$. Natural compound (dihydromyricetin or berberine) treatment reduces hepatic IR injury by enhancing the SIRT-1/FOXO3a-induced autophagy pathway; FOXO3a siRNA or SIRT-1 inhibition diminishes the protective effect of these compounds ${ }^{27,57}$. SIRT-1 activation also protects against pulmonary emphysema via deacetylation of FOXO-3a and reduction of premature senescence in mice ${ }^{58}$. Here, we found that nobiletin treatment increased SIRT-1 and FOXO3a expression, inducing autophagy during hepatic IR injury, and this protective effect against hepatic IR was abolished by SIRT-1 inhibition. These data indicate that activation of autophagy via the SIRT-1/FOXO3a pathway is crucial for nobiletin-mediated protection. However, further epigenetic studies are required to determine the mechanism of action of nobiletin regarding the regulation of SIRT-1 expression, either through direct interaction or indirectly.

FOXO3a is phosphorylated by the serine/threonine protein kinase AKT and becomes sequestered by $14-3-3$ proteins in the cytoplasm, which inhibits FOXO3a transcriptional activity $^{28}$. Ethanol treatment decreases the levels of phosphorylated AKT and FOXO3a, which in turn increases FOXO3-mediated transcription of autophagyrelated genes. However, deletion of the farnesoid $\mathrm{X}$ receptor impairs FOXO3a-mediated autophagy and exacerbates alcohol-induced liver injury. Resveratrol, a SIRT-1 agonist, enhances ethanol-induced expression of autophagy-related genes through FOXO3a deacetylation $^{30,56}$. Here, nobiletin increased SIRT-1 and FOXO3a expression and FOXO3a nuclear translocation, simultaneously reducing phosphorylation of AKT and FOXO3a in the cytosol and resulting in FOXO3a-mediated autophagy induction. We hypothesize that nobiletin attenuates IR-induced liver damage by enhancing autophagy through the PI3K/AKT/FOXO3a and SIRT-1/ FOXO3a pathways.

Our results indicate that nobiletin may contribute to epigenetic control of the SIRT-1 promoter and/or transacting factors to upregulate SIRT-1 expression. Similarly, resveratrol, a specific SIRT-1 agonist, has been shown to upregulate SIRT-1 expression and protect against cerebral IR injury, radiation-induced intestinal injury, and sepsisinduced myocardial injury ${ }^{59-61}$. In addition to natural polyphenols such as resveratrol, recent studies have identified small molecule SIRT-1 activators with enhanced potency, such as imidazothiazoles (e.g., SRT1720) and molecules bearing benzimidazole and ureabased scaffolds ${ }^{62}$. These SIRT-1 activators enhance the substrate affinity through an allosteric mechanism, particularly through a conserved glutamate (E230) in the $\mathrm{N}$ terminal activation domain of SIRT-1, and facilitate formation or stabilization of the activated SIRT-1 conformation ${ }^{63}$. Apparently, the structure of nobiletin is not consistent with these identified SIRT-1 activators, and the crystal structure of the nobiletin-enzyme complex has not yet been revealed, unlike resveratrol or other SIRT-1 activators $^{64,65}$. We speculate that nobiletin may stabilize an enzyme-substrate interaction to activate SIRT-1 in a FOXO3a substrate-specific manner by strengthening the binding of "loose-binding" substrates, similar to the action of resveratrol on $\mathrm{p} 53^{66}$. Since the effect of nobiletin was abolished by EX-527, nobiletin potentially acts by enhancing SIRT-1 enzymatic activity; however, further characterization is required to determine the specific molecular mechanisms of nobiletin.

Nobiletin is metabolized by cytochrome P450 enzymes, and the resulting metabolites exert different biological 
and pharmacological activities ${ }^{67}$. Nobiletin is a promising candidate for drug development, but the target receptors need to be verified so that nobiletin can be used precisely for clinical applications. Recent studies have identified several metabolites of nobiletin with biological activities, including 3',4'-didemethylnobiletin, 5-demethylnobiletin, and 4'-demethylnobiletin. These metabolites exhibit significant and often greater effects than nobiletin with respect to the reduction of inflammation, oxidative stress, carcinogenesis, and atherogenesis ${ }^{33}$. The nobiletin metabolites that enhance autophagy and/or mitochondrial function have yet to be studied; however, the aforementioned metabolites could be promising candidates and could facilitate the identification of the specific target receptors of nobiletin.

In summary, this study is the first to demonstrate that nobiletin protects against hepatic IR injury through the induction of autophagy and homeostatic control of mitochondrial biogenesis and dynamics. These effects occur through SIRT-1/FOXO3a-mediated autophagy and SIRT-1/PGC-1 $\alpha$-mediated mitochondrial regulation.

\section{Acknowledgements}

This study was supported by the Basic Science Research Program through the National Research Foundation (NRF) of Korea funded by the Ministry of Science, ICT and Future Planning (NRF-2015R1A5A2008833 and NRF2017R1D1A1B03035634).

\section{Conflict of interest}

The authors declare that they have no conflict of interest.

\section{Publisher's note}

Springer Nature remains neutral with regard to jurisdictional claims in published maps and institutional affiliations.

Supplementary information accompanies this paper at https://doi.org/ 10.1038/s12276-019-0245-z.

Received: 8 October 2018 Revised: 7 January 2019 Accepted: 8 January 2019.

Published online: 26 April 2019

\section{References}

1. Weigand, K. et al. Ischemia/Reperfusion injury in liver surgery and transplantation: pathophysiology. HPB Surg. 2012, 176723 (2012).

2. Zhai, Y., Petrowsky, H., Hong, J. C., Busuttil, R. W. \& Kupiec-Weglinski, J. W. Ischaemia-reperfusion injury in liver transplantation--from bench to bedside. Nat. Rev. Gastroenterol. Hepatol. 10, 79-89 (2013).

3. Kalogeris, T., Bao, Y. \& Korthuis, R. J. Mitochondrial reactive oxygen species: a double edged sword in ischemia/reperfusion vs preconditioning. Redox Biol. 2 , 702-714 (2014)

4. Peralta, C., Jimenez-Castro, M. B. \& Gracia-Sancho, J. Hepatic ischemia and reperfusion injury: effects on the liver sinusoidal milieu. J. Hepatol. 59 1094-1106 (2013).

5. Klionsky, D. J. \& Emr, S. D. Autophagy as a regulated pathway of cellular degradation. Science 290, 1717-1721 (2000).

6. Czaja, M. J. et al. Functions of autophagy in normal and diseased liver. Autophagy 9, 1131-1158 (2013).

7. $\mathrm{Xu}, \mathrm{D}$. et al. The triterpenoid CDDO-imidazolide ameliorates mouse liver ischemia-reperfusion injury through activating the $\mathrm{Nrf2} / \mathrm{HO}-1$ pathway enhanced autophagy. Cell Death Dis. 8, e2983 (2017).
8. Yun, N., Cho, H. I. \& Lee, S. M. Impaired autophagy contributes to hepatocellular damage during ischemia/reperfusion: heme oxygenase-1 as a possible regulator. Free Radic. Biol. Med 68, 168-177 (2014).

9. Lemasters, J. J., Theruvath, T. P., Zhong, Z. \& Nieminen, A. L. Mitochondrial calcium and the permeability transition in cell death. Biochim Biophys. Acta 1787, 1395-1401 (2009).

10. Lu, Y. et al. Asiatic acid ameliorates hepatic ischemia/reperfusion injury in rats via mitochondria-targeted protective mechanism. Toxicol. Appl. Pharm. 338, 214-223 (2018)

11. Khader, A. et al. Sirtuin 1 stimulation attenuates ischemic liver injury and enhances mitochondrial recovery and autophagy. Crit. Care Med. 44, e651-e663 (2016)

12. Bordone, L. \& Guarente, L. Calorie restriction, SIRT1 and metabolism: understanding longevity. Nat. Rev. Mol. Cell Biol. 6, 298-305 (2005).

13. Nemoto, S., Fergusson, M. M. \& Finkel, T. SIRT1 functionally interacts with the metabolic regulator and transcriptional coactivator PGC-1\{alpha\}. J. Biol. Chem. 280, 16456-16460 (2005).

14. Li, X. SIRT1 and energy metabolism. Acta. Biochim. Biophys. Sin. (Shanghai) 45, 51-60 (2013).

15. Si, H. \& Liu, D. Dietary antiaging phytochemicals and mechanisms associated with prolonged survival. J. Nutr. Biochem. 25, 581-591 (2014).

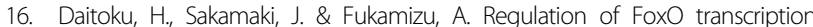
factors by acetylation and protein-protein interactions. Biochim. Biophys. Acta. 1813, 1954-1960 (2011).

17. Tang, B. L. Sirt1 and the mitochondria. Mol. Cells 39, 87-95 (2016).

18. Malik, S. et al. Nobiletin ameliorates cisplatin-induced acute kidney injury due to its anti-oxidant, anti-inflammatory and anti-apoptotic effects. Exp. Toxicol. Pathol. 67, 427-433 (2015).

19. Zhang, L. et al. Nobiletin promotes antioxidant and anti-inflammatory responses and elicits protection against ischemic stroke in vivo. Brain Res. 1636, 130-141 (2016)

20. Aoki, K., Yokosuka, A., Mimaki, Y., Fukunaga, K. \& Yamakuni, T. Nobiletin induces inhibitions of Ras activity and mitogen-activated protein kinase kinase/extracellular signal-regulated kinase signaling to suppress cell proliferation in C6 rat glioma cells. Biol. Pharm. Bull. 36, 540-547 (2013).

21. Umeno, A., Horie, M., Murotomi, K. Nakajima, Y. \& Yoshida, Y. Antioxidative and antidiabetic effects of natural polyphenols and isoflavones. Molecules 21, E708 (2016).

22. $\mathrm{Wu}, \mathrm{X}$. et al. Nobiletin attenuates adverse cardiac remodeling after acute myocardial infarction in rats via restoring autophagy flux. Biochem. Biophys. Res. Commun. 492, 262-268 (2017).

23. $\mathrm{Wu}, \mathrm{Y}$. et al. Nobiletin ameliorates ischemia-reperfusion injury by suppressing the function of Kupffer cells after liver transplantation in rats. Biomed. Pharm. 89, 732-741 (2017).

24. Suzuki, S. et al. The beneficial effect of a prostaglandin 12 analog on ischemic rat liver. Transplantation 52, 979-983 (1991).

25. Austin, S. \& St-Pierre, J. PGC1alpha and mitochondrial metabolism--emerging concepts and relevance in ageing and neurodegenerative disorders. J. Cell Sci. 125, 4963-4971 (2012).

26. Shin, J. K. \& Lee, S. M. Genipin protects the liver from ischemia/reperfusion injury by modulating mitochondrial quality control. Toxicol. Appl. Pharm. 328, 25-33 (2017).

27. Lin, $Y$. et al. Berberine protects against ischemia/reperfusion injury after orthotopic liver transplantation via activating Sirt1/FoxO3alpha induced autophagy. Biochem. Biophys. Res. Commun. 483, 885-891 (2017).

28. Nho, R. S. \& Hergert, P. FoxO3a and disease progression. World J. Biol. Chem. 5, 346-354 (2014).

29. Yang, C. et al. Cyclic helix B peptide inhibits ischemia reperfusion-induced renal fibrosis via the PI3K/Akt/FoxO3a pathway. J. Transl. Med. 13, 355 (2015).

30. Ni, H. M., Du, K., You, M. \& Ding, W. X. Critical role of FoxO3a in alcohol-induced autophagy and hepatotoxicity. Am. J. Pathol. 183, 1815-1825 (2013).

31. Zhao, J. et al. FoxO3 coordinately activates protein degradation by the autophagic/lysosomal and proteasomal pathways in atrophying muscle cells. Cell Metab. 6, 472-483 (2007).

32. Kalogeris, T., Baines, C. P., Krenz, M. \& Korthuis, R. J. Cell biology of ischemia/ reperfusion injury. Int Rev. Cell Mol. Biol. 298, 229-317 (2012).

33. Huang, $\mathrm{H}$. et al. The multifunctional effects of nobiletin and its metabolites in vivo and in vitro. Evid. Based Complement Altern. Med. 2016, 2918796 (2016).

34. Noguchi, S., Atsumi, H., Iwao, Y., Kan, T. \& Itai, S. Nobiletin: a citrus flavonoid displaying potent physiological activity. Acta Crystallogr C. Struct. Chem. 72 124-127 (2016) 
35. Klein, T. \& Bischoff, R. Physiology and pathophysiology of matrix metalloproteases. Amino Acids 41, 271-290 (2011).

36. Coito, A. J. Leukocyte transmigration across endothelial and extracellular matrix protein barriers in liver ischemia/reperfusion injury. Curr. Opin. Organ Transpl. 16, 34-40 (2011).

37. Duarte, S., Baber, J., Fujii, T. \& Coito, A. J. Matrix metalloproteinases in liver injury, repair and fibrosis. Matrix Biol. 44-46, 147-156 (2015).

38. Kato, H., Duarte, S., Liu, D., Busuttil, R. W. \& Coito, A. J. Matrix metalloproteinase2 (MMP-2) gene deletion enhances MMP-9 activity, impairs PARP-1 degradation, and exacerbates hepatic ischemia and reperfusion injury in mice. PLoS One 10, e0137642 (2015).

39. Hamada, T., Fondevila, C., Busuttil, R. W. \& Coito, A. J. Metalloproteinase-9 deficiency protects against hepatic ischemia/reperfusion injury. Hepatology 47, 186-198 (2008).

40. Tiriveedhi, V. et al. Protective role of bortezomib in steatotic liver ischemia/ reperfusion injury through abrogation of MMP activation and YKL-40 expression. Transpl. Immunol. 30, 93-98 (2014).

41. Jassem, W. \& Heaton, N. D. The role of mitochondria in ischemia/reperfusion injury in organ transplantation. Kidney Int. 66, 514-517 (2004).

42. Crompton, M. The mitochondrial permeability transition pore and its role in cell death. Biochem. J. 341(Pt 2), 233-249 (1999).

43. Jornayvaz, F. R. \& Shulman, G. I. Regulation of mitochondrial biogenesis. Essays Biochem. 47, 69-84 (2010).

44. Joe, Y. et al. Cilostazol attenuates murine hepatic ischemia and reperfusion injury via heme oxygenase-dependent activation of mitochondrial biogenesis. Am. J. Physiol. Gastrointest. Liver Physiol. 309, G21-G29 (2015).

45. Lagouge, $M$. et al. Resveratrol improves mitochondrial function and protects against metabolic disease by activating SIRT1 and PGC-1alpha. Cell 127, 1109-1122 (2006).

46. Chen, H. \& Chan, D. C. Emerging functions of mammalian mitochondrial fusion and fission. Hum. Mol. Genet. 2, R283-R289 (2005). 14 Spec No.

47. Chen, $Y$. et al. Mitofusin 2 protects hepatocyte mitochondrial function from damage induced by GCDCA. PLoS One 8, e65455 (2013).

48. Yang, $X$. et al. Inhibition of Drp1 protects against senecionine-induced mitochondria-mediated apoptosis in primary hepatocytes and in mice. Redox Biol. 12, 264-273 (2017).

49. Li, J. et al. Tumour necrosis factor-alpha promotes liver ischaemia-reperfusion injury through the PGC-1alpha/Mfn2 pathway. J. Cell Mol. Med. 18, 1863-1873 (2014).

50. Li, Y. et al. Peroxisome proliferator-activated receptor delta regulates mitofusin 2 expression in the heart. J. Mol. Cell Cardiol. 46, 876-882 (2009).

51. Soriano, F. X. et al. Evidence for a mitochondrial regulatory pathway defined by peroxisome proliferator-activated receptor-gamma coactivator-1 alpha, estrogen-related receptor-alpha, and mitofusin 2. Diabetes 55, 1783-1791 (2006).

52. Wang, J. H., Behrns, K. E., Leeuwenburgh, C. \& Kim, J. S. Critical role of autophage in ischemia/reperfusion injury to aged livers. Autophagy $\mathbf{8}, 140-141$ (2012).

53. Sun, $\mathrm{K}$. et al. Autophagy lessens ischemic liver injury by reducing oxidative damage. Cell Biosci. 3, 26 (2013).

54. Wang, J. H. et al. Autophagy suppresses age-dependent ischemia and reperfusion injury in livers of mice. Gastroenterology 141, 2188-2199.e6 (2011).

55. Xiong, X., Tao, R., DePinho, R. A. \& Dong, X. C. The autophagy-related gene 14 (Atg14) is regulated by forkhead box $O$ transcription factors and circadian rhythms and plays a critical role in hepatic autophagy and lipid metabolism. J. Biol. Chem. 287, 39107-39114 (2012).

56. Manley, S. et al. Farnesoid X receptor regulates forkhead Box O3a activation in ethanol-induced autophagy and hepatotoxicity. Redox Biol. 2, 991-1002 (2014).

57. Chen, Y. et al. Dihydromyricetin protects against liver ischemia/reperfusion induced apoptosis via activation of FOXO3a-mediated autophagy. Oncotarget 7. 76508-76522 (2016).

58. Yao, H. et al. SIRT1 protects against emphysema via FOXO3-mediated reduction of premature senescence in mice. J. Clin. Invest. 122, 2032-2045 (2012).

59. An, R. et al. Resveratrol alleviates sepsisinduced myocardial injury in rats by suppressing neutrophil accumulation, the induction of TNFalpha and myocardial apoptosis via activation of Sirt1. Mol. Med. Rep. 14, 5297-5303 (2016).

60. He, Q. et al. Resveratrol alleviates cerebral ischemia/reperfusion injury in rats by inhibiting NLRP3 inflammasome activation through Sirt1-dependent autophagy induction. Int Immunopharmacol. 50, 208-215 (2017).

61. Zhang, $\mathrm{H}$. et al. The protective effects of Resveratrol against radiation-induced intestinal injury. BMC Complement Alter. Med. 17, 410 (2017).

62. Hubbard, B. P. \& Sinclair, D. A. Small molecule SIRT1 activators for the treatment of aging and age-related diseases. Trends Pharm. Sci. 35, 146-154 (2014).

63. Hubbard, B. P. et al. Evidence for a common mechanism of SIRT1 regulation by allosteric activators. Science 339, 1216-1219 (2013).

64. Cao, D. et al. Structural basis for allosteric, substrate-dependent stimulation of SIRT1 activity by resveratrol. Genes Dev. 29, 1316-1325 (2015).

65. Dai, H. et al. Crystallographic structure of a small molecule SIRT1 activatorenzyme complex. Nat. Commun. 6, 7645 (2015).

66. Hou, X., Rooklin, D., Fang, H. \& Zhang, Y. Resveratrol serves as a proteinsubstrate interaction stabilizer in human SIRT1 activation. Sci. Rep. 6, 38186 (2016).

67. Koga, N. et al. In vitro metabolism of nobiletin, a polymethoxy-flavonoid, by human liver microsomes and cytochrome P450. Xenobiotica 41, 927-933 (2011). 